Arkivoc

Archive for

Organic Chemistry
The Free Internet Journal

for Organic Chemistry
Paper

Arkivoc 2017, part iii, 9-20

\title{
Application of four-membered ring chalcogenation reagents to the synthesis of new phosphorus-chalcogen heterocycles
}

\author{
Guoxiong Hua, David B. Cordes, Alexandra M. Z. Slawin, and J. Derek Woollins* \\ EaStCHEM School of Chemistry, University of St Andrews, Fife, KY16 9ST, U.K. \\ Email:jdw3@st-and.ac.uk
}

Dedicated to Prof. Oleg A. Rakitin on the occasion of his 65th birthday

Received 07-20-2016

Accepted 08-16-2016

Published on line $08-29-2016$

\section{Abstract}

The reaction of four-membered ring chalcogenation reagents such as Lawesson's reagent, 2,4-diferrocenyl1,3,2,4-diathiadiphosphetane 2,4-disulfide (the ferrocene analogy of Lawesson's reagent) and Woollins' reagent with alkyl- or aryl-dithiols in refluxing toluene gave a series of five- to seven-membered organophosphorus-chalcogen heterocycles in $24 \%$ to $87 \%$ yields. Five representative X-ray structures confirm the formation of these five- to seven-membered heterocycles.
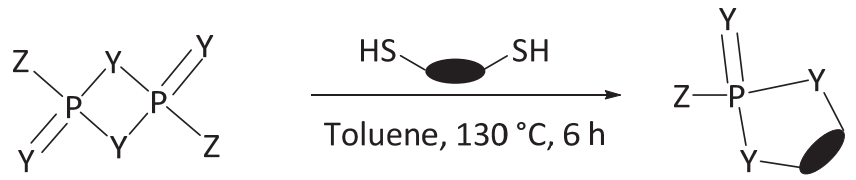

$\mathrm{Y}=\mathrm{S}$, Se

$\mathrm{Z}=$ phenyl, 4-methoxyphenyl, ferrocenyl

$=\mathrm{CH}_{2} \mathrm{CH}_{2}, \mathrm{CH}_{2} \mathrm{CH}_{2} \mathrm{CH}_{2}, \mathrm{CH}_{2} \mathrm{CH}_{2} \mathrm{CH}_{2} \mathrm{CH}_{2}$, $1,2-\mathrm{C}_{6} \mathrm{H}_{4}, 4-\mathrm{CH}_{3}-1,2-\mathrm{C}_{6} \mathrm{H}_{3}$

Keywords: Lawesson's reagent; Woollins' reagent, phosphorus-chalcogen heterocycles, chalcogenation; dithiols 


\section{Introduction}

The chemistry of organophosphorus-chalcogen heterocycles has attracted extensive attention for several decades. Heterocycles with small ring systems have found many applications both as useful starting materials in the synthesis of more elaborate structures and as valuable targets of organic and inorganic synthesis. ${ }^{1} \mathrm{~A}$ variety of organophosphorus-chalcogen heterocycles including three- to ten-membered ring systems has been developed due to their wide-ranging applications in synthetic chemistry. ${ }^{2-5}$ The most important $\mathrm{RP} / \mathrm{S}(\mathrm{Se})$ heterocycles are the four-membered ring compounds, Lawesson's reagent ( $L R, R=p-\mathrm{C}_{6} \mathrm{H}_{4} \mathrm{OMe}$ ), a highly efficient thionation reagent, ${ }^{6-11}$ 2,4-diferrocenyl-1,3,2,4-diathiadiphosphetane 2,4-disulfide (FCLR, a ferrocene analogy of Lawesson's reagent), another alternatively efficient thionation reagent, ${ }^{12-16}$ and 2,4-diphenyl-1,3diselenadiphosphetane 2,4-diselenide $\left[\left\{\mathrm{PhP}(\mathrm{Se})(\mu \text {-Se) }\}_{2}\right]\right.$ (Woollins' reagent, WR, $\mathrm{R}=\mathrm{Ph}$, a selenium counterpart of Lawesson's reagent), as a highly efficient selenation reagent. ${ }^{17-25}$ Very recently, we have reported these chalcogenation reagents used as efficient building blocks for the synthesis of a series of phosphorus-chalcogen macrocycles incorporating two phosphorus atoms and four chalcogen atoms $\left[X_{2}-P_{2}-X_{2}\right.$ $(X=0, S, S e)]$ in the central ring. ${ }^{26-28}$ In order to enrich further the library of organo phosphorus-chalcogen heterocycles, herein we report the preparation of a series of small organo phosphorus-chalcogen heterocycles from the reaction of chalcogenation reagents (LR, FCLR and WR) with alkyl-dithiol or aryl-dithiols, and five representative $\mathrm{X}$-ray structures.

\section{Results and Discussion}

Treating Lawesson's reagent with two molar equivalents of alkyl- or aryl-dithiol in refluxing toluene gave the corresponding five- and six-membered ring heterocycles $1-3$ in $45 \%$ to $65 \%$ yields, respectively, as shown in Scheme 1. Heterocycles 1-3 were obtained as white solids or pastes in good yields, and are soluble in normal organic solvents such as dichloromethane, chloroform, THF, acetonitrile, acetone and so on. All of the above heterocycles were found to be air-stable both as solids and in solution. The identity of 1-3 was determined through a combination of mass spectrometric (El or $\mathrm{Cl}$ ) techniques, and NMR $\left({ }^{1} \mathrm{H},{ }^{13} \mathrm{C}\right.$ and $\left.{ }^{31} \mathrm{P}\right)$ spectroscopy. In all cases mass spectrometry found the expected $[\mathrm{M}]^{+}$or $[\mathrm{M}+\mathrm{H}]^{+}$. The ${ }^{1} \mathrm{H}$ and ${ }^{13} \mathrm{C}$ spectra of $1-3$ show clearly the presence of both the aromatic and alkoxy substituents displaying the expected coupling constants. Singlets at 71.4, 64.7 and $88.4 \mathrm{ppm}$ were observed respectively in their ${ }^{31} \mathrm{P}\left\{{ }^{1} \mathrm{H}\right\} \mathrm{NMR}$ spectra. Attempts to prepare ring systems of more than six-members from the reaction of $\mathbf{L R}$ with longer-chain $\left[\left(-\mathrm{CH}_{2}-\mathrm{I}_{n}, \mathrm{n}>3\right]\right.$ dithiol precursors failed and always led to very messy products.

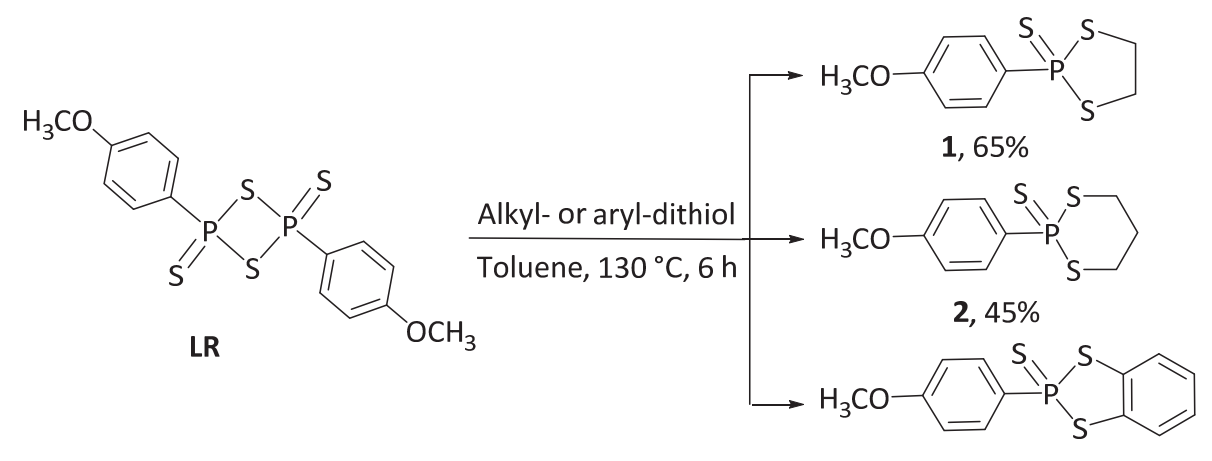

$3,58 \%$

Scheme 1. Synthesis of phosphorus-sulfur heterocycles 1-3 from LR and alkyl- or aryl-diols. 
The X-ray structures of heterocycles 1-3 reveal (Figures 1-3 and Tables 1 and 2) that all compounds crystallize with one independent molecule in the unit cell. 1-3 adopt puckered conformations with 1 being a pseudo-chair arrangement and 2,3 being open envelope conformations. Compared to the structures of $\mathbf{2}$ and $\mathbf{3}$, the structure of $\mathbf{1}$ is highly symmetrical with the mean plane of newly formed ring being perpendicular to the aryl ring. In comparison, the mean planes of the newly formed ring and the aryl ring in $\mathbf{2}$ and $\mathbf{3}$ are rotated with the dihedral angles of $87.70^{\circ}$ and $80.98^{\circ}$, respectively. In the newly formed ring the central phosphorus atom lies $0.195 \AA$ in $1,0.349 \AA$ in 2 and $0.332 \AA$ in 3 out of the P-S-S- $C_{n}(n=2,3)$ mean plane. The $P=S$ double bond lengths in the structures of 1-3 are 1.934(2) $\AA, 1.9390(10) \AA$ and 1.9351(7) $\AA$, respectively, cf dithiophosphono disulfides $[1.9203(14)-1.9303(7) \AA]]^{29-31}$ are slightly shorter than that in other terminal phosphine sulphide bond lengths in the Cambridge Crystallographic Database (average $1.965 \AA$ ). ${ }^{32}$
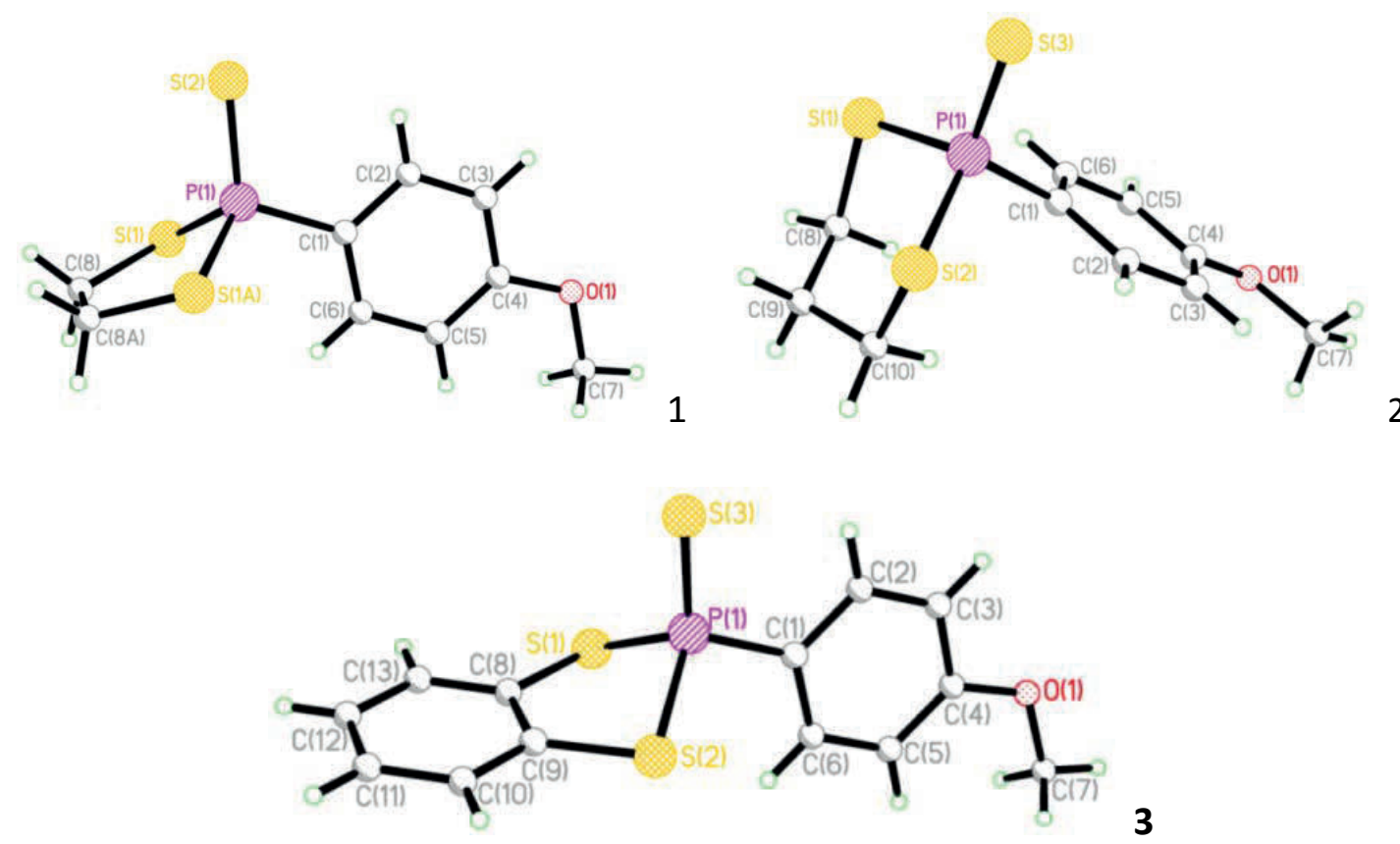

Figure 1. Single crystal X-ray structures of compounds 1, 2 and 3.

The reaction of WR and alkyl- or aryl-dithiol was carried out under identical condition. Phosphorusselenium heterocycles 4-6 were obtained by the reaction of WR with the corresponding alkyl-dithiols in good to excellent yields (Scheme 3). Meanwhile, the reaction of WR with two equivalents of aryl-dithiol afforded as the sole product seven-membered ring heterocycle 7 in very low yield (24\%). The results indicated that the stability of heterocycles is affected by the replacement of sulfur by selenium in the phosphorus center; the $\mathrm{P}(\mathrm{Se})$-containing heterocycles seem to be more stable than those with a $\mathrm{P}(\mathrm{S})$ motif. Furthermore, the building block with $\pi$-system such as phenyl ring is less favorable than building block such as linear $\mathrm{CH}_{2} \mathrm{CH}_{2} \mathrm{CH}_{2} \mathrm{CH}_{2}$ chain system. Two diastereoisomers were found in ca. $2: 1$ intensity ratio for seven-membered heterocycle 6 due to its highly twisted newly formed ring. In all cases mass spectrometry found the expected $[\mathrm{M}]^{+}$or $[\mathrm{M}+\mathrm{H}]^{+}$, with the matching isotope distributions as the expected patterns. The ${ }^{1} \mathrm{H}$ and ${ }^{13} \mathrm{C}$ spectra of heterocycles $4-7$ confirm the presence of phenyl ring showing the expected coupling constants. The ${ }^{31} \mathrm{P} N M R$ spectra of heterocycles 4-7 display sharp singlets at 73.1, 46.2, 70.5 (65.3) and 70.6 ppm, respectively, and each signal is accompanied by one set of selenium satellites $(816 \mathrm{~Hz}$ for $\mathbf{4}, 786 \mathrm{~Hz}$ for 5,808 and $789 \mathrm{~Hz}$ for $\mathbf{6}$, and $833 \mathrm{~Hz}$ for 7), indicating that in each compound there is a $\mathrm{P}=\mathrm{Se}$ double bond present. This is further substantiated by the 
${ }^{77}$ Se NMR spectra, which display a doublet with matching coupling constants, indicating the presence of a $\mathrm{P}=\mathrm{Se}$ double bond in each compound.

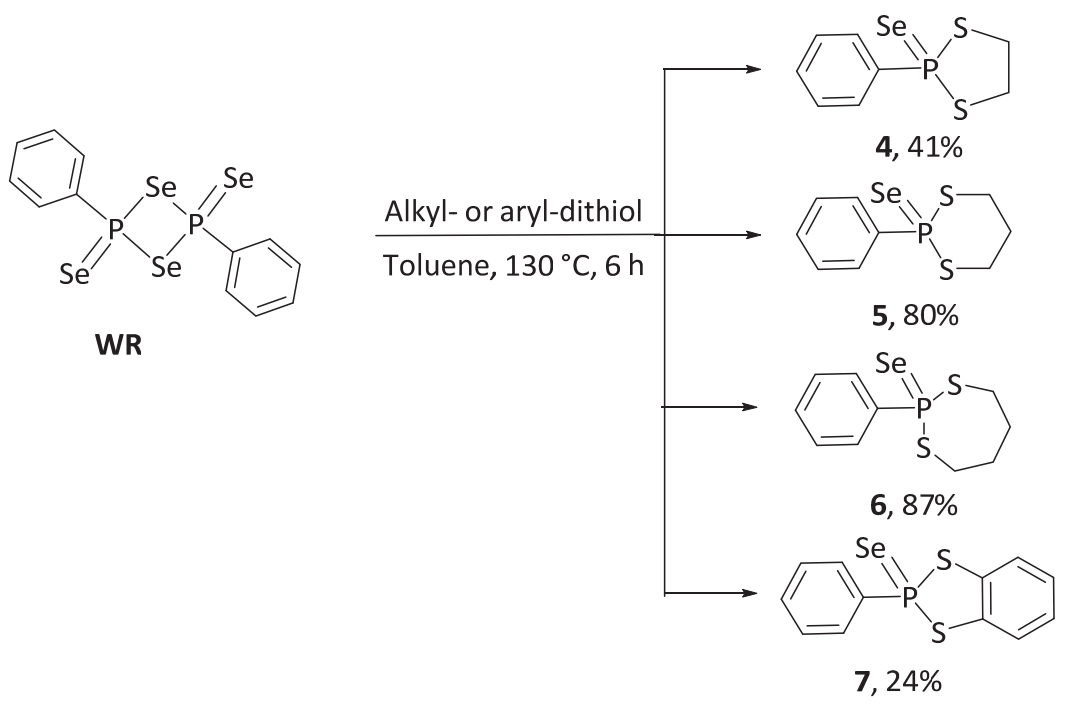

Scheme 2. Synthesis of phosphorus-sulfur-selenium heterocycles 4-7 from WR and alkyl- or aryl-dithiols.

Analogous reactions were carried out by using $\operatorname{FCLR}^{29,30}$ and dithiols. The reaction of FcLR with two molar equivalents of alkyl- or aryl-dithiols in refluxing toluene afforded five or seven-membered ring heterocycles 810 in good yields as shown in Scheme 2. The yields suggest that the ring size of the newly formed ring plays a key role in the formation of heterocyclic compounds 8-10, the formation of five-membered rings 8 and 10 are favoured compared the formation of seven-membered ring $\mathbf{9}$. Though the ${ }^{31} \mathrm{P} N M R$ spectrum of the reaction mixture did suggest the formation of the corresponding six-membered ring heterocycle $\left(\delta_{P}=71.9 \mathrm{ppm}\right)$, the reaction of FCLR with 1,3-propane-dithiol did not lead to any isolatable pure product. The heterocycles 8-10 were found to be air stable both as solids and in solution. Two signals were found in $c a .3: 2$ intensity ratio for seven-membered heterocycle 9 and we speculate that there are conformational isomers present with selenium/phenyl substituents being able to occupy axial or equatorial positions though we have not conducted VT NMR experiments. ${ }^{1} \mathrm{H}$ NMR and ${ }^{13} \mathrm{C}$ NMR spectra of compounds 8-10 were as expected confirming the presence of all the characteristic peaks of the ferrocene backbones. The ${ }^{31} \mathrm{P} N M R$ spectra of 810 exhibit sharp singlets in the range of $\delta=81.4$ to $93.6 \mathrm{ppm}$, the values are consistent with those in heterocycles 1-3.

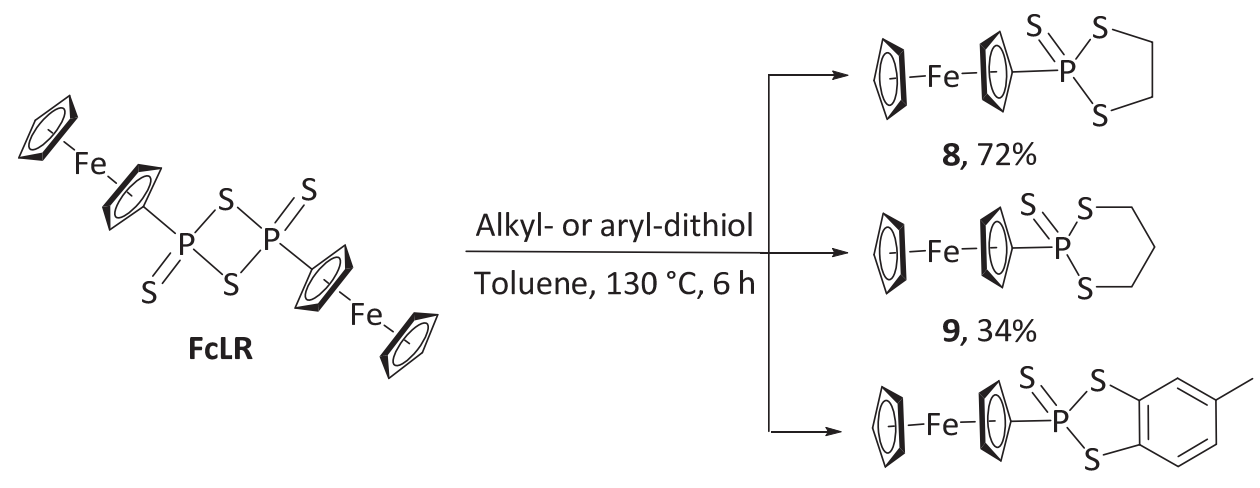

$10,76 \%$

Scheme 3. Synthesis of phosphorus-sulfur heterocycles 8-10 from FcLR and alkyl-or aryl-dithiols. 
The X-ray structures of $\mathbf{5}$ and $\mathbf{1 0}$ were obtained and show that the $\mathrm{PhP}=\mathrm{X}(\mathrm{X}=\mathrm{S}$ or $\mathrm{Se})$ is attached across the dithiol in a newly formed five-membered $\mathrm{C}_{2} \mathrm{PS}_{2}$ ring or six-membered $\mathrm{C}_{3} \mathrm{PS}_{2}$ ring as shown in Figure 2 and Tables 1 and 2. Compound $\mathbf{1 0}$ crystallizes with one independent molecule within the unit cell; compound $\mathbf{5}$ crystallizes with two independent molecules within the unit cell. Both structures adopt the envelope like conformation, having very similar structural motif to the structure of $\mathbf{3}$. The newly formed five-membered ring $\mathrm{P}(\mathrm{S})-\mathrm{S}_{2}-\mathrm{C}_{2}$ in 10 is nearly planar with a mean deviation of $0.1522 \AA$; however, the newly formed six-membered ring $\mathrm{P}(\mathrm{Se})-\mathrm{S}_{2}-\mathrm{C}_{3}$ in 5 is highly puckered. The P-S single bond lengths in 10 [2.1045(14) and 2.1048(14) $\AA$ ] are comparable to the P-S single bond lengths in 3 [2.1028(8) and 2.1038(7) $\AA$ ], but considerably longer than the corresponding P-S single bond lengths in 1 [2.0899(17) $\AA$ ], 2 [2.0724(9) and 2.0665(9) $\AA$ ] and in the similar fivemembered $\mathrm{P}(\mathrm{S}) \mathrm{S}_{2} \mathrm{C}_{2}$ ring structures: for example, 2,5-dithia-1-phenyl-1-thiophosphorus(V)-cyclopentane [2.087 $\AA],^{33} 4,5$-diphenyl-2-ferrocenyl-1,3,2-dithiaphosphane 2-sulfide[2.095(2) and 2.089(2) $\AA$ ], ${ }^{34}$ 2-t-butyl-2-thioxo1,3,2-dithiaphospholane [2.096(1) and 2.081(1) $\AA]$ and 2-(3,5-dimethylphenyl)-2-thioxo-1,3,2dithiaphospholane $[2.084(1)$ and $2.078(1) \AA],{ }^{35}$ but is considerably shorter than the corresponding P-S single bond lengths in 2-(1,1-dimethylethyl)-2-sulfide-4,5-[1,2-dicarbacloso-dodecaborano(12)]-1,3,2dithiaphospholane $[2.1335(16)$ and $2.1429(17) \AA]$; ${ }^{36}$ meanwhile, the $P=S$ double bond [1.9233(17) $\AA$ ] is substantially shorter than that in 1-3 [1.934(2) to 1.9390(10) $\AA$ ] and in the similar structures [1.932(1) $1.938(2) \AA] .{ }^{33-36}$ The plane of the phenyl ring is almost perpendicular $\left[87.98^{\circ}\right]$ to the $C_{p}$ ring attached to phosphorus atom. The P-S single bond distances in 5 [2.0688(10) - 2.0793(11) Å] are consistent with those in 2 [2.0793(11) and 2.0665(9) $\AA$ ]. However, they are marginally shorter than the P-S single bond distances in 1, 3 $[2.0899(17)-2.1038(7) \AA]$ and in similar structures $[2.081(1)-2.1429(17) \AA] .{ }^{33-36}$ The $P=S e$ double bond distances in 5 [2.1020(9) [2.1011(8)] $\AA$ ] are close to the $P=S e$ double bond distances in the five-membered ring $\mathrm{P}(\mathrm{Se}) \mathrm{C}_{4}$ systems such as $1,1^{\prime}$-bis(2,5-dimethylphospholanyl)ferrocene- $P, P^{\prime} 1,1^{\prime}$-diselenide [avg. $2.1066 \AA$ ] and

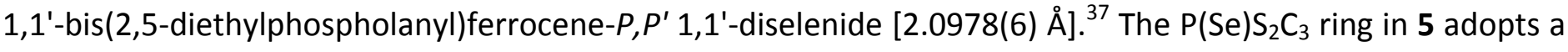
chair geometry with the substituent phenyl ring in an axial site.

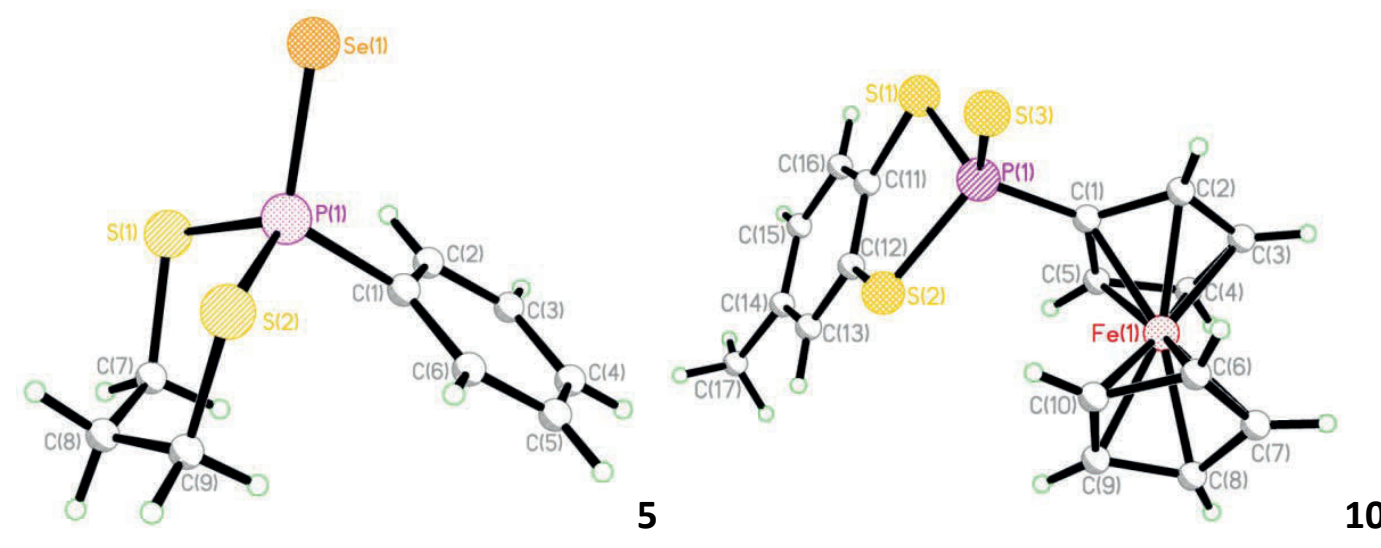

Figure 2. Single crystal X-ray structures of compounds 5 and 10. 
Table 1. Details of the X-ray data collections and refinements for compounds $1,2,3,5$ and 10

\begin{tabular}{cccccc}
\hline Compound & $\mathbf{1}$ & $\mathbf{2}$ & $\mathbf{3}$ & $\mathbf{5}$ & $\mathbf{1 0}$ \\
\hline Formula & $\mathrm{C}_{9} \mathrm{H}_{11} \mathrm{OPS}_{3}$ & $\mathrm{C}_{10} \mathrm{H}_{13} \mathrm{OPS}_{3}$ & $\mathrm{C}_{13} \mathrm{H}_{11} \mathrm{OPS}_{3}$ & $\mathrm{C}_{9} \mathrm{H}_{11} \mathrm{PS}_{2} \mathrm{Se}$ & $\mathrm{C}_{17} \mathrm{H}_{15} \mathrm{FePS}_{3}$ \\
$M$ & 262.34 & 276.37 & 310.38 & 293.24 & 402.31 \\
Crystal system & Orthorhombic & Monoclinic & Triclinic & Monoclinic & Monoclinic \\
Space group & $P n m a$ & $P 2_{1} / c$ & $P-1$ & $P 2_{1} / n$ & $P 2_{1} / n$ \\
$a / \AA$ & $20.961(7)$ & $11.526(2)$ & $7.6496(18)$ & $11.287(6)$ & $12.407(5)$ \\
$b / \AA$ & $6.993(3)$ & $11.228(2)$ & $8.2387(13)$ & $6.901(2)$ & $9.349(3)$ \\
$c / \AA$ & $8.166(3)$ & $10.470(2)$ & $11.274(2)$ & $20.754(7)$ & $15.176(8)$ \\
$\alpha$ & 90 & 90 & $75.0145(10)$ & 90 & 90 \\
$b$ & 90 & $113.301(5)$ & $89.379(10)$ & $108.245(9)$ & $108.086(12)$ \\
$V$ & 90 & 90 & $84.850(11)$ & 90 & 90 \\
$U / A^{3}$ & $1197.0(4)$ & $1244.5(4)$ & $683.5(2)$ & $2351.4(13)$ & $1673.3(12)$ \\
$Z$ & 4 & 4 & 2 & 8 & 4 \\
$\mu / m m^{-1}$ & 7.178 & 6.945 & 6.419 & 36.388 & 13.617 \\
Reflections collected & 7313 & 19224 & 11162 & 36172 & 13116 \\
Independent & 1183 & 2279 & 2493 & 4294 & 3077 \\
reflections & & & & & 0.0828 \\
$R_{\text {int }}$ & 0.0300 & 0.1766 & 0.0279 & 0.0280 & 0.0393 \\
$R 1$ & 0.0709 & 0.0449 & 0.0226 & 0.0653 & 0.0794 \\
\hline$R 2[I>2 \sigma(I)]$ & 0.1893 & 0.1430 & 0.0831 & &
\end{tabular}

Table 2. Selected bond lengths ( $(\AA)$ and angles $\left({ }^{\circ}\right)$ for the structures of 1-3, $\mathbf{5}$ and 10

\begin{tabular}{cccccc}
\hline & $\mathbf{1}$ & $\mathbf{2}$ & $\mathbf{3}$ & $\mathbf{5}$ & $\mathbf{1 0}$ \\
\hline $\mathrm{P}(1)-\mathrm{S}(1)$ & $2.0899(17)$ & $2.0724(9)$ & $2.1028(8)$ & $2.0712(11)[2.0793(11)]$ & $2.1045(14)$ \\
$\mathrm{P}(1)-\mathrm{S}(2)$ & & $2.0665(9)$ & $2.1038(7)$ & $2.0703(12)[2.0688(10)]$ & $2.1048(14)$ \\
$\mathrm{P}(1)-\mathrm{X}(3)$ & $1.934(2)$ & $1.9390(10)$ & $1.9351(7)$ & $2.1020(9)[2.1011(8)]^{*}$ & $1.9233(17)$ \\
$\mathrm{P}(1)-\mathrm{C}(1)$ & & $1.804(3)$ & $1.7917(19)$ & $1.816(2)[1.818(3)]$ & $1.773(3)$ \\
$\mathrm{S}(1)-\mathrm{P}(1)-\mathrm{S}(2)$ & $98.90(8)$ & $106.14(4)$ & $95.90(3)$ & $106.86(4)[106.10(4)]$ & $97.86(6)$ \\
$\mathrm{S}(1)-\mathrm{P}(1)-\mathrm{X}(3)$ & & $110.11(5)$ & $115.51(3)$ & $110.16(4)[109.08(4)]^{*}$ & $112.87(6)$ \\
$\mathrm{S}(2)-\mathrm{P}(1)-\mathrm{X}(3)$ & $115.05(7)$ & $110.98(4)$ & $115.74(3)$ & $110.46(4)[111.15(4)]^{*}$ & $115.70(6)$ \\
$\mathrm{X}(3)-\mathrm{P}(1)-\mathrm{C}(1)$ & $113.3(2)$ & $114.25(8)$ & $114.15(6)$ & $114.90(9)[116.46(8)]^{*}$ & $113.80(13)$ \\
$\mathrm{S}(1)-\mathrm{P}(1)-\mathrm{C}(1)$ & $106.58(13)$ & $107.29(8)$ & $108.12(7)$ & $106.03(8)[107.49(7)]$ & $107.91(12)$ \\
$\mathrm{S}(2)-\mathrm{P}(1)-\mathrm{C}(1)$ & & $107.68(9)$ & $105.55(6)$ & $108.04(9)[106.01(8)]$ & $107.32(12)$ \\
\hline
\end{tabular}

$* \mathrm{X}=\mathrm{S}$ or $\mathrm{Se}$. 
In summary, we have successfully developed an efficient route for synthesis of a series of organo phosphorus-chalcogen heterocycles via the reaction of four-membered ring thionation reagent, 2,4-bis(4methoxyphenyl)-1,3,2,4-dithiadiphosphetane 2,4-disulfide (LR, Lawesson's reagent) or 2,4-diferrocenyl1,3,2,4-diathiadiphosphetane 2,4-disulfide (FCLR, a ferrocene analogue of Lawesson's reagent) and 2,4bis(phenyl)-1,3-diselenadiphosphethane-2,4-diselenide $\left[\{\mathrm{PhP}(\mathrm{Se})(\mu \text {-Se })\}_{2}\right]$ (Woollins' reagent, WR, R = Ph, a selenium counterpart of Lawesson's reagent) with alkenyl-dithiols or aryl-dithiols in refluxing toluene solution. Five representative X-ray structures are discussed to confirm the formation of these heterocycles. It is anticipated that this route would be a convenient pathway to synthesize novel macrocyclic compounds. This method allows P-S or P-Se heterocycles to be easily available for further investigations into their chemistry and biological properties.

\section{Experimental Section}

General. Unless otherwise stated, all reactions were carried out under an oxygen free nitrogen atmosphere using pre-dried solvents and standard Schlenk techniques; subsequent chromatographic and work up procedures were performed in air. All commercially available reagents including alkyldithiols and aryldithiols were used as supplied without further purification unless stated otherwise. ${ }^{1} \mathrm{H}(400.1 \mathrm{MHz}),{ }^{13} \mathrm{C}(100.6 \mathrm{MHz})$, ${ }^{31} \mathrm{P}-\left\{{ }^{1} \mathrm{H}\right\}(162.0 \mathrm{MHz})$ and ${ }^{77} \mathrm{Se}-\left\{{ }^{1} \mathrm{H}\right\}\left(51.5 \mathrm{MHz}\right.$ referenced to external $\left.\mathrm{Me}_{2} \mathrm{Se}\right) \mathrm{NMR}$ spectra were recorded at 25 ${ }^{\circ} \mathrm{C}$ (unless stated otherwise). IR spectra were recorded as $\mathrm{KBr}$ pellets in the range of $4000-250 \mathrm{~cm}^{-1}$. Mass spectrometry ( $\mathrm{m} / \mathrm{z}, \mathrm{HRMS}$ ) data was performed using either atmospheric pressure chemical ionization (APCI) or electron ionization (EI) using a TOM mass analyzer.

The X-ray crystal structure for compound 1 was collected at $173 \mathrm{~K}$ using a Rigaku MM007 High brilliance RA generator/confocal optics and Mercury CCD system, and the structures for compounds 2, 3, 5 and 10 were collected using a Rigaku FR-X Ultrahigh brilliance Microfocus RA generator/confocal optics and Rigaku XtaLAB P200 system, both with Mo K $\alpha$ radiation $(\lambda=0.71075 \AA)$. Intensity data were collected using both $\omega$ and $\phi$ steps $(\mathbf{1})$, or $\omega$ steps alone $(\mathbf{2}, \mathbf{3}, \mathbf{5}, \mathbf{1 0})$, accumulating area detector images spanning at least a hemisphere of reciprocal space. All data were corrected for Lorentz polarization effects. Absorption effects were corrected on the basis of multiple equivalent reflections or by semi-empirical methods using CrystalClear. ${ }^{38}$ Structures were solved by charge-flipping (Superflip: 1), ${ }^{39}$ direct (SIR-2011: 2, 3, 10) ${ }^{40}$ or Patterson (PATTY: 5) ${ }^{41}$ methods and refined by full-matrix least-squares against $F^{2}$ (SHELXTL). ${ }^{42}$ Non-hydrogen atoms were refined anisotropically, and hydrogen atoms were assigned riding isotropic displacement parameters and constrained to idealized geometries. All calculations were performed using the CrystalStructure ${ }^{43}$ interface. These data can be obtained free of charge via www.ccdc.cam.ac.uk/conts/retrieving.html or from the Cambridge Crystallographic Data Centre, 12 Union Road, Cambridge CB2 1EZ, UK; fax (+44) 1223-336-033; e-mail: deposit@ccdc.cam.ac.uk. CCDC Nos 1494991-1494996.

General Procedure for Preparation of Heterocycles 1-10. A white or brown suspension of LR or FCLR or WR $(1.0 \mathrm{mmol})$ and alkyl- or aryl-dithiol $(2.0 \mathrm{mmol})$ in dry toluene $(20 \mathrm{~mL})$ was heated at $130{ }^{\circ} \mathrm{C}$ for $6 \mathrm{~h}$. Upon cooling to room temperature and removing unreacted solid the filtrate was dried in vacuo and the residue was dissolved in dichloromethane $(c a .2 \mathrm{~mL}$ ) and loaded onto a silica gel column (1:1 hexane/dichloromethane or dichloromethane as eluent) to give compounds 1-10.

2-(4-Methoxyphenyl)-1,3,2-dithiaphospholane 2-sulfide (1). White solid (65\% yield). Selected IR $\left(\mathrm{KBr}, \mathrm{cm}^{-1}\right)$ : 1434(m), 1414(m), 1279(m), 1201(m), 1088(s), 995(m), 936(m), 745(s), 686(s), 566(vs), 528(s), 472(s). ${ }^{1} \mathrm{H} N M R$ $\left(\mathrm{CD}_{2} \mathrm{Cl}_{2}, \delta\right), 7.98(\mathrm{dd}, J(\mathrm{P}, \mathrm{H})=20.7 \mathrm{~Hz}, J(\mathrm{H}, \mathrm{H})=8.0 \mathrm{~Hz}, 2 \mathrm{H}), 6.91(\mathrm{dd}, J(\mathrm{P}, \mathrm{H})=7.6 \mathrm{~Hz}, J(\mathrm{H}, \mathrm{H})=8.0 \mathrm{~Hz}, 2 \mathrm{H}), 3.78(\mathrm{~s}$, 
3H), 3.72-3.53 (m, 4H) ppm. ${ }^{13} \mathrm{C} N M R\left(\mathrm{CD}_{2} \mathrm{Cl}{ }_{2}, \delta\right), 164.6(\mathrm{~d}, J(\mathrm{P}, \mathrm{C})=105 \mathrm{~Hz}), 135.2,129.4,114.7,56.5,43.9$ ppm. ${ }^{31} \mathrm{P} N M R\left(\mathrm{CD}_{2} \mathrm{Cl}_{2}, \delta\right), 71.4$ ppm. Mass spectrum $\left(\mathrm{El}^{+}, \mathrm{m} / z\right), 262[\mathrm{M}]^{+}$. Accurate mass measurement [El ${ }^{+}$, $m / z]: 261.9707[\mathrm{M}]^{+}$, calculated mass for $\mathrm{C}_{9} \mathrm{H}_{11} \mathrm{OPS}_{3}: 261.9710$.

2-(4-Methoxyphenyl)-1,3,2-dithiaphosphinane 2-sulfide (2). White paste (45\% yield). Selected IR $\left(\mathrm{KBr}, \mathrm{cm}^{-1}\right)$ : 1588(s), 1562(m), 1493(s), 1461(m), 1419(m), 1289(m), 1256(s), 1179(s), 1095(s), 1016(s), 831(s), 799(m), 677(s), 637(m), 622(m), 561(s), 509(s). ${ }^{1} \mathrm{H} N M R\left(\mathrm{CDCl}_{3}, \delta\right), 8.12(\mathrm{dd}, J(\mathrm{P}, \mathrm{H})=14.6 \mathrm{~Hz}, J(\mathrm{H}, \mathrm{H})=8.0 \mathrm{~Hz}, 2 \mathrm{H}), 7.05$ $(\mathrm{dd}, J(\mathrm{P}, \mathrm{H})=3.2 \mathrm{~Hz}, J(\mathrm{H}, \mathrm{H})=8.0 \mathrm{~Hz}, 2 \mathrm{H}), 3.89(\mathrm{~s}, 3 \mathrm{H}), 3.59-3.50(\mathrm{~m}, 2 \mathrm{H}), 3.15-3.04(\mathrm{~m}, 2 \mathrm{H}), 2.29-2.07(\mathrm{~m}, 2 \mathrm{H})$ ppm. ${ }^{13} \mathrm{C} \mathrm{NMR}\left(\mathrm{CDCl}_{3}, \delta\right), 163.4(\mathrm{~d}, J(\mathrm{P}, \mathrm{C})=3.5 \mathrm{~Hz}), 133.2(\mathrm{~d}, J(\mathrm{P}, \mathrm{C})=14.0 \mathrm{~Hz}), 124.7(\mathrm{~d}, J(\mathrm{P}, \mathrm{C})=93.3 \mathrm{~Hz}), 114.5$ $(d, J(P, C)=15.6 \mathrm{~Hz}), 55.6,31.1(\mathrm{~d}, J(\mathrm{P}, \mathrm{C})=3.4 \mathrm{~Hz}), 25.8(\mathrm{~d}, J(\mathrm{P}, \mathrm{C})=4.7 \mathrm{~Hz}) \mathrm{ppm} .{ }^{31} \mathrm{P} \mathrm{NMR}\left(\mathrm{CDCl}_{3}, \delta\right), 64.7 \mathrm{ppm}$. Mass spectrum $\left(\mathrm{Cl}^{+}, \mathrm{m} / \mathrm{z}\right), 277[\mathrm{M}+\mathrm{H}]^{+}$. Accurate mass measurement $\left[\mathrm{Cl}^{+}, \mathrm{m} / \mathrm{z}\right]: 276.9940[\mathrm{M}+\mathrm{H}]^{+}$, calculated mass for $\mathrm{C}_{10} \mathrm{H}_{13} \mathrm{OPS}_{3} \mathrm{H}: 276.9939$.

2-(4-Methoxyphenyl)-1,3,2-benzodihydrodithiaphosphole 2-sulfide (3). White paste (58\% yield). Selected IR $\left(\mathrm{KBr}, \mathrm{cm}^{-1}\right):$ 1589(s), 1494(m), 1444(s), 1295(m), 1262(s), 1181(m), 1096(s), 1021(s), 928(m), 839(s), 800(m), 748(s), 691(s), 624(s), 540(s), 514(s), 396(m). ${ }^{1} \mathrm{H} N M R\left(\mathrm{CDCl}_{3}, \delta\right), 8.23(\mathrm{dd}, J(\mathrm{P}, \mathrm{H})=15.3 \mathrm{~Hz}, J(\mathrm{H}, \mathrm{H})=8.0 \mathrm{~Hz}, 2 \mathrm{H})$, 7.45-7.37 (m, 2H), 7.24-7.16 (m, 2H), 7.10-7.00 (m, 2H), $3.89(\mathrm{~s}, 3 \mathrm{H}) \mathrm{ppm} .{ }^{13} \mathrm{C} \mathrm{NMR}\left(\mathrm{CDCl}_{3}, \delta\right), 163.6(\mathrm{~d}, J(\mathrm{P}, \mathrm{C})=$ $3.5 \mathrm{~Hz}), 134.2(\mathrm{~d}, J(\mathrm{P}, \mathrm{C})=14.8 \mathrm{~Hz}), 131.2,130.3(\mathrm{~d}, J(\mathrm{P}, \mathrm{C})=92.9 \mathrm{~Hz}), 127.3,126.8,125.9(\mathrm{~d}, J(\mathrm{P}, \mathrm{C})=8.8 \mathrm{~Hz})$, 55.7 ppm. ${ }^{31} \mathrm{P} \mathrm{NMR}\left(\mathrm{CDCl}_{3}, \delta\right), 88.4 \mathrm{ppm}$. Mass spectrum $\left(\mathrm{Cl}^{+}, \mathrm{m} / \mathrm{z}\right), 311[\mathrm{M}+\mathrm{H}]^{+}$. Accurate mass measurement $\left[\mathrm{Cl}^{+}, \mathrm{m} / \mathrm{z}\right]: 310.9784[\mathrm{M}+\mathrm{H}]^{+}$, calculated mass for $\mathrm{C}_{13} \mathrm{H}_{11} \mathrm{OPS}_{3} \mathrm{H}: 310.9782$.

2-Phenyl-1,3,2-dithiaphospholane 2-selenide (4). Pale orange paste (41\% yield). Selected $\mathrm{IR}\left(\mathrm{KBr}, \mathrm{cm}^{-1}\right)$ : 1590(s), 1496(s), 1404(m), 1303(m), 1264(s), 1186(s), 1121(m), 1096(s), 1020(m), 922(s), 821(m), 747(m), 686(s), 531(m), 437(m). ${ }^{1} \mathrm{H}$ NMR $\left(\mathrm{CD}_{2} \mathrm{Cl} 2, \delta\right), 8.07-7.99(\mathrm{~m}, 2 \mathrm{H})$, 7.44-7.40 (m, 3H), 3.74-3.50 (m, 4H) ppm. ${ }^{13} \mathrm{C}$ $\operatorname{NMR}\left(\mathrm{CD}_{2} \mathrm{Cl}_{2}, \delta\right), 138.5(\mathrm{~d}, J(\mathrm{P}, \mathrm{C})=101.5 \mathrm{~Hz}), 133.0(\mathrm{~d}, J(\mathrm{P}, \mathrm{C})=3.1 \mathrm{~Hz}), 132.0(\mathrm{~d}, J(\mathrm{P}, \mathrm{C})=13.0 \mathrm{~Hz}), 129.3(\mathrm{~d}$, $J(P, C)=14.3 \mathrm{~Hz}), 44.8)$ ppm. ${ }^{31} \mathrm{P} N M R\left(\mathrm{CD}_{2} \mathrm{Cl}_{2}, \delta\right), 73.1(\mathrm{~s}, J(\mathrm{P}=\mathrm{Se})=816 \mathrm{~Hz}) \mathrm{ppm} .{ }^{77} \mathrm{Se} \mathrm{NMR}\left(\mathrm{CD}_{2} \mathrm{Cl}_{2}, \delta\right), 389.5(\mathrm{~d}$, $\left.{ }^{1} J(\mathrm{P}=\mathrm{Se})=816 \mathrm{~Hz}\right)$ ppm. Mass spectrum $\left(\mathrm{EI}^{+}, \mathrm{m} / \mathrm{z}\right), 275.9(20 \%), 276.9(19 \%), 277.9(49 \%), 279.9(100 \%)$, 281.9(27\%) $[\mathrm{M}]^{+}$. Accurate mass measurement $\left[\mathrm{El}^{+}, \mathrm{m} / \mathrm{z}\right]: 279.9045[\mathrm{M}]^{+}$, calculated mass for $\mathrm{C}_{8} \mathrm{H}_{9} \mathrm{PS}_{2} \mathrm{Se}$ 279.9048.

2-Phenyl-1,3,2-dithiaphosphinane 2-selenide (5). Pale pink solid (80\% yield). M.p. 100-102 ${ }^{\circ} \mathrm{C}$. Selected IR $\left(\mathrm{KBr}, \mathrm{cm}^{-1}\right):$ 1475(w), 1431(s), 1415(m), 1265(s), 1175(m), 1089(s), 997(s), 895(m), 857(s), 743(s), 689(s), 615(s), 551(vs), 492(s), 329(m). ${ }^{1} \mathrm{H}$ NMR ( $\left.\mathrm{CDCl}_{3}, \delta\right), 8.21-8.15(\mathrm{~m}, 2 \mathrm{H}), 7.60-7.54(\mathrm{~m}, 3 \mathrm{H}), 3.44-3.34(\mathrm{~m}, 2 \mathrm{H}), 3.08-2.98$ $(\mathrm{m}, 2 \mathrm{H}), 2.27-2.12(\mathrm{~m}, 2 \mathrm{H}) \mathrm{ppm} .{ }^{13} \mathrm{C} \mathrm{NMR}\left(\mathrm{CDCl}_{3}, \delta\right), 133.1(\mathrm{~d}, J(\mathrm{P}, \mathrm{C})=73.9 \mathrm{~Hz}), 132.9(\mathrm{~d}, J(\mathrm{P}, \mathrm{C})=3.6 \mathrm{~Hz}), 131.1$ $(d, J(P, C)=12.0 \mathrm{~Hz}), 129.2(\mathrm{~d}, J(\mathrm{P}, \mathrm{C})=14.1 \mathrm{~Hz}), 31.9(\mathrm{~d}, J(\mathrm{P}, \mathrm{C})=3.7 \mathrm{~Hz}), 25.3(\mathrm{~d}, J(\mathrm{P}, \mathrm{C})=5.2 \mathrm{~Hz}) \mathrm{ppm} .{ }^{31} \mathrm{P} \mathrm{NMR}$ $\left(\mathrm{CDCl}_{3}, \delta\right), 46.2(\mathrm{~s}, J(\mathrm{P}, \mathrm{Se})=786 \mathrm{~Hz})$ ppm. ${ }^{77} \mathrm{Se} \mathrm{NMR}\left(\mathrm{CDCl}_{3}, \delta\right),-27.3(\mathrm{~d}, J(\mathrm{P}, \mathrm{Se})=786 \mathrm{~Hz})$ ppm. Mass spectrum $\left(\mathrm{El}^{+}, \mathrm{m} / \mathrm{z}\right), 290.9(18 \%), 291.9(16 \%), 292.9(48 \%), 294.9(100 \%), 296.9(21 \%)\left[\mathrm{M}^{+}\right.$. Accurate mass measurement $\left[\mathrm{Cl}^{+}, \mathrm{m} / \mathrm{z}\right]: 294.9275[\mathrm{M}+\mathrm{H}]^{+}$, calculated mass for $\mathrm{C}_{9} \mathrm{H}_{11} \mathrm{PS}_{2} \mathrm{SeH}: 294.9276$.

2-Phenyl-1,3,2-dithiaphosphepane 2-selenide (6). Milky paste (87\% yield). Two diastereoisomers were found in ca. 2 : 1 intensity ratio in multi-NMR spectra. Selected IR ( KBr, cm $\left.{ }^{-1}\right): 1541(\mathrm{~m}), 1434(\mathrm{~s}), 1303(\mathrm{~s}), 1092(\mathrm{~s})$, 746(s), 687(s), 617(m), 575(s), 528(s), 475(s), 352(m), 279(s). ${ }^{1} \mathrm{H}$ NMR $\left(\mathrm{CDCl}_{3}, \delta\right), 8.22(\mathrm{~m}, 4 \mathrm{H}), 7.59-7.48(\mathrm{~m}$, $6 \mathrm{H}), 3.40-3.20(\mathrm{~m}, 2 \mathrm{H}), 2.98-2.89(\mathrm{~m}, 2 \mathrm{H}), 2.60-2.47(\mathrm{~m}, 4 \mathrm{H}), 2.38-2.11(\mathrm{~m}, 2 \mathrm{H}), 1.85-1.60(\mathrm{~m}, 2 \mathrm{H}), 1.44-1.32(\mathrm{~m}$, 2H) ppm. ${ }^{13} \mathrm{C} \mathrm{NMR}\left(\mathrm{CDCl}_{3}, \delta\right), 135.5(\mathrm{~d}, J(\mathrm{P}, \mathrm{C})=74.3 \mathrm{~Hz}), 134.0(\mathrm{~d}, J(\mathrm{P}, \mathrm{C})=76.5 \mathrm{~Hz}), 132.7(\mathrm{~d}, J(\mathrm{P}, \mathrm{C})=3.7 \mathrm{~Hz})$, $132.4(\mathrm{~d}, J(\mathrm{P}, \mathrm{C})=3.5 \mathrm{~Hz}), 131.3(\mathrm{~d}, J(\mathrm{P}, \mathrm{C})=12.5 \mathrm{~Hz}), 131.1(\mathrm{~d}, J(\mathrm{P}, \mathrm{C})=12.5 \mathrm{~Hz}), 35.0(\mathrm{~d}, J(\mathrm{P}, \mathrm{C})=3.3 \mathrm{~Hz}), 33.1(\mathrm{~d}$, $J(P, C)=3.8 \mathrm{~Hz}), 32.7,32.6,30.4,28.4(\mathrm{~d}, J(\mathrm{P}, \mathrm{C})=5.0 \mathrm{~Hz}), 24.1,24.0 \mathrm{ppm} .{ }^{31} \mathrm{P} \mathrm{NMR}\left(\mathrm{CDCl}_{3}, \delta\right), 70.5(\mathrm{~s}, J(\mathrm{P}, \mathrm{Se})=$ $808 \mathrm{~Hz}), 65.3(\mathrm{~s}, J(\mathrm{P}, \mathrm{Se})=789 \mathrm{~Hz}) \mathrm{ppm} .{ }^{77} \mathrm{Se} \mathrm{NMR}\left(\mathrm{CDCl}_{3}, \delta\right),-10.7(\mathrm{~d}, J(\mathrm{P}, \mathrm{Se})=789 \mathrm{~Hz}),-139.6(\mathrm{~d}, J(\mathrm{P}, \mathrm{Se})=808$ $\mathrm{Hz}$ ) ppm. Mass spectrum (El $\left.\mathrm{I}^{+} \mathrm{m} / \mathrm{z}\right), 304.9(19 \%), 305.9(17 \%), 306.9(47 \%), 308.9(100 \%), 310.9(19 \%)[\mathrm{M}]^{+}$. Accurate mass measurement $\left[\mathrm{Cl}^{+}, \mathrm{m} / \mathrm{z}\right]: 308.9433[\mathrm{M}+\mathrm{H}]^{+}$, calculated mass for $\mathrm{C}_{10} \mathrm{H}_{13} \mathrm{PS}_{2} \mathrm{SeH}: 308.9433$. 
2-Phenyl-1,3,2-benzodithiaphosphole 2-selenide (7). Pale red paste (24\% yield). Selected $\mathrm{IR}\left(\mathrm{KBr}, \mathrm{cm}^{-1}\right)$ : 1440(s), 1303(m), 1260(m), 1090(s), 1027(m), 802(m), 748(s), 705(m), 687(m), 617(m), 573(s), 535(m), 480(m), 454(m), 421(m). ${ }^{1} \mathrm{H}$ NMR $\left(\mathrm{CDCl}_{3}, \delta\right), 8.23(\mathrm{dd}, J(\mathrm{P}, \mathrm{H})=16.6 \mathrm{~Hz}, J(\mathrm{H}, \mathrm{H})=8.3 \mathrm{~Hz}, 2 \mathrm{H}), 7.59-7.49(\mathrm{~m}, 3 \mathrm{H}), 7.40(\mathrm{dd}$, $J(P, H)=3.2 \mathrm{~Hz}, J(\mathrm{H}, \mathrm{H})=6.3 \mathrm{~Hz}, 2 \mathrm{H}), 7.21(\mathrm{~d}, J(\mathrm{H}, \mathrm{H})=6.3 \mathrm{~Hz}, 2 \mathrm{H}) \mathrm{ppm} .{ }^{13} \mathrm{C} \mathrm{NMR}\left(\mathrm{CDCl}_{3}, \delta\right), 135.8,135.5(\mathrm{~d}, J(\mathrm{P}, \mathrm{C})$ $=69.1 \mathrm{~Hz}), 133.0(\mathrm{~d}, J(\mathrm{P}, \mathrm{C})=3.6 \mathrm{~Hz}), 131.4(\mathrm{~d}, J(\mathrm{P}, \mathrm{C})=13.1 \mathrm{~Hz}), 128.6(\mathrm{~d}, J(\mathrm{P}, \mathrm{C})=14.6 \mathrm{~Hz}), 127.4,125.6(\mathrm{~d}$, $J(P, C)=8.3 \mathrm{~Hz})$ ppm. ${ }^{31} \mathrm{P} \mathrm{NMR}\left(\mathrm{CDCl}_{3}, \delta\right), 70.6(\mathrm{~s}, J(\mathrm{P}, \mathrm{Se})=833 \mathrm{~Hz}) \mathrm{ppm} .{ }^{77} \mathrm{Se} \mathrm{NMR}\left(\mathrm{CDCl}_{3}, \delta\right),-13.3(\mathrm{~d}, J(\mathrm{P}, \mathrm{Se})=$ $834 \mathrm{~Hz}$ ) ppm. Mass spectrum (El $\left.{ }^{+}, \mathrm{m} / \mathrm{z}\right), 323.9(18 \%), 324.9 .9(17 \%), 325.9(49 \%), 327.9(100 \%), 329.9(20 \%)[\mathrm{M}]^{+}$. Accurate mass measurement $\left[\mathrm{Cl}^{+}, \mathrm{m} / \mathrm{z}\right]: 328.9122[\mathrm{M}+\mathrm{H}]^{+}$, calculated mass for $\mathrm{C}_{12} \mathrm{H}_{9} \mathrm{PS}_{2} \mathrm{SeH}: 328.9126$.

2-Ferrocenyl-1,3,2-dithiaphospholane 2-sulfide (8). Pale yellow solid (72\% yield). Selected $\mathrm{IR}\left(\mathrm{KBr}, \mathrm{cm}^{-1}\right)$ : 1411(m), 1368(m), 1190(s), 1107(m), 1030(m), 939(vs), 892(s), 823(m), 794(m), 754(s), 669(s), 488(m), 468(s),

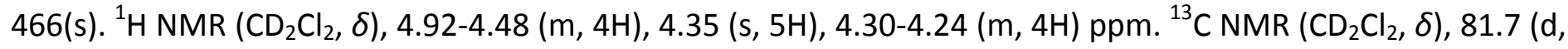
$J(P, C)=130.4 \mathrm{~Hz})$, ), $73.8(\mathrm{~d}, J(\mathrm{P}, \mathrm{C})=21.4 \mathrm{~Hz})$, ), $73.6(\mathrm{~d}, J(\mathrm{P}, \mathrm{C})=16.5 \mathrm{~Hz}), 71.5$, ), $43.6(\mathrm{~d}, J(\mathrm{P}, \mathrm{C})=34.3 \mathrm{~Hz}) \mathrm{ppm}$.

${ }^{31} \mathrm{P}$ NMR $\left(\mathrm{CD}_{2} \mathrm{Cl}_{2}, \delta\right), 93.6$ ppm. Mass spectrum $\left(\mathrm{El}^{+}, \mathrm{m} / \mathrm{z}\right), 330[\mathrm{M}]^{+}$. Accurate mass measurement $\left[\mathrm{El}^{+}, \mathrm{m} / \mathrm{z}\right]$ : 329.9261 [M] ${ }^{+}$, calculated mass for $\mathrm{C}_{12} \mathrm{H}_{13} \mathrm{FePS}_{3}$ : 329.9266 .

2-Ferrocenyl-1,3,2-dithiaphosphepane 2-sulfide (9). Yellow paste (34\% yield). Two diastereoisomers were found in ca. $3: 2$ intensity ratio in multi-NMR spectra. Selected IR $\left(\mathrm{KBr}, \mathrm{cm}^{-1}\right): 1433(\mathrm{~s}), 1409(\mathrm{~m}), 1282(\mathrm{~m})$, 1238(m), 1172(s), 1104(m), 1024(s), 944(m), 826(s), 677(vs), 525(s), 486(s). ${ }^{1} \mathrm{H}$ NMR (CDCl,$\left.\delta\right), 4.75-4.64(\mathrm{~m}$, $4 \mathrm{H}), 4.58-4.51(\mathrm{~m}, 4 \mathrm{H}), 4.40(\mathrm{~s}, 5 \mathrm{H}), 4.38(\mathrm{~s}, 5 \mathrm{H}), 3.35-2.90(\mathrm{~m}, 8 \mathrm{H}), 2.61-2.53(\mathrm{~m}, 2 \mathrm{H}), 2.27-2.11(\mathrm{~m}, 2 \mathrm{H}), 1.97-$ $1.72(\mathrm{~m}, 4 \mathrm{H}) \mathrm{ppm} .{ }^{13} \mathrm{C} N M R\left(\mathrm{CDCl}_{3}, \delta\right), 80.6(\mathrm{~d}, J(\mathrm{P}, \mathrm{C})=101.1 \mathrm{~Hz}), 78.4(\mathrm{~d}, J(\mathrm{P}, \mathrm{C})=101.3 \mathrm{~Hz}), 72.3(\mathrm{~d}, J(\mathrm{P}, \mathrm{C})=$ $11.9 \mathrm{~Hz}), 72.2(\mathrm{~d}, J(\mathrm{P}, \mathrm{C})=15.1 \mathrm{~Hz}), 71.7(\mathrm{~d}, J(\mathrm{P}, \mathrm{C})=11.7 \mathrm{~Hz}), 71.3(\mathrm{~d}, J(\mathrm{P}, \mathrm{C})=14.8 \mathrm{~Hz}), 70.8,70.7,34.7(\mathrm{~d}, J(\mathrm{P}, \mathrm{C})$ $=4.1 \mathrm{~Hz}), 33.6(\mathrm{~d}, J(\mathrm{P}, \mathrm{C})=4.7 \mathrm{~Hz}), 24.1(\mathrm{~d}, J(\mathrm{P}, \mathrm{C})=3.6 \mathrm{~Hz}), 24.0(\mathrm{~d}, J(\mathrm{P}, \mathrm{C})=4.4 \mathrm{~Hz}) \mathrm{ppm} .{ }^{31} \mathrm{P} \mathrm{NMR}(\mathrm{CDCl}, \delta)$, 81.7 and 81.4 ppm. Mass spectrum $\left(\mathrm{Cl}^{+}, \mathrm{m} / z\right), 369[\mathrm{M}+\mathrm{H}]^{+}$. Accurate mass measurement $\left[\mathrm{Cl}^{+}, \mathrm{m} / z\right]: 368.9649$ $[\mathrm{M}+\mathrm{H}]^{+}$, calculated mass for $\mathrm{C}_{14} \mathrm{H}_{17} \mathrm{FePS}_{3} \mathrm{H}: 368.9652$.

2-Ferrocenyl-4-methyl-1,3,2-benzodithiaphosphole 2-sulfide (10). Yellow solid (76\% yield). M.p. $121-123^{\circ} \mathrm{C}$. Selected IR ( $\left.\mathrm{KBr}, \mathrm{cm}^{-1}\right):$ 1585(m), 1457(s), 1409(m), 1387(m), 1175(s), 1118(m), 1022(s), 908(m), 824(s), 731(s), 693(vs), 682(vs), 536(s), 487(s), 450(m), 525(m). ${ }^{1} \mathrm{H} N M R\left(\mathrm{CDCl}_{3}, \delta\right), 7.30(\mathrm{~s}, 1 \mathrm{H}), 7.24-7.23(\mathrm{~m}, 1 \mathrm{H}), 7.03-7.01$ $(\mathrm{m}, 1 \mathrm{H}), 4.82-4.79(\mathrm{~m}, 2 \mathrm{H}), 4.60-4.59(\mathrm{~m}, 2 \mathrm{H}), 4.42(\mathrm{~s}, 5 \mathrm{H}), 2.35(\mathrm{~s}, 3 \mathrm{H}) \mathrm{ppm} .{ }^{13} \mathrm{C} \mathrm{NMR}\left(\mathrm{CDCl}_{3}, \delta\right), 137.3,134.5$, 131.1, 128.0, $126.5(\mathrm{~d}, J(\mathrm{P}, \mathrm{C})=8.1 \mathrm{~Hz}), 125.7(\mathrm{~d}, J(\mathrm{P}, \mathrm{C})=8.1 \mathrm{~Hz}), 76.9(\mathrm{~d}, J(\mathrm{P}, \mathrm{C})=99.4 \mathrm{~Hz}), 73.4(\mathrm{~d}, J(\mathrm{P}, \mathrm{C})=16.3$ $\mathrm{Hz}), 73.4(\mathrm{~d}, J(\mathrm{P}, \mathrm{C})=12.7 \mathrm{~Hz}), 70.9,21.1 \mathrm{ppm} .{ }^{31} \mathrm{P} \mathrm{NMR}\left(\mathrm{CDCl}_{3}, \delta\right), 89.5 \mathrm{ppm}$. Mass spectrum $\left(\mathrm{Cl}^{+}, \mathrm{m} / \mathrm{z}\right), 402$ $[\mathrm{M}+\mathrm{H}]^{+}$. Accurate mass measurement $\left[\mathrm{Cl}^{+}, \mathrm{m} / \mathrm{z}\right]: 401.9412[\mathrm{M}+\mathrm{H}]^{+}$, calculated mass for $\mathrm{C}_{17} \mathrm{H}_{14} \mathrm{FePS}_{3} \mathrm{H}$ : 401.9417.

\section{Acknowledgements}

We are grateful to the University of St Andrews for financial support and the EPSRC National Mass Spectrometry Service Centre (Swansea) for mass spectral measurements.

\section{References}

1. Rotstein, B. H.; Zaretsky, S.; Rai, V.; Yudin, A. K. Chem. Rev. 2014, 114, 8323-8359. http://dx.doi.org/10.1021/cr400615v

2. Chivers, T.; Manners, I. Inorganic rings and polymers of the p-block elements: from fundamentals to applications. RSC Publishing: Cambridge, U. K., 2009, pp 256-259. 
3. Woollins, J. D.; Laitinen, R. S. Selenium and tellurium chemistry: from small molecules to biomolecules and materials. Springer, London, 2011, pp 1-40.

http://dx.doi.org/10.1007/978-3-642-20699-3

4. Timperley, C. M. Best synthetic methods: Organophosphorus (V) chemistry. Academic Press, London, 2015, pp 633-720.

5. Lecher, H. Z.; Greenwood, R. A.; Whitehouse, K. C.; Chao, T. H. J. Am. Chem. Soc. 1956, 78, 5018-5023. http://dx.doi.org/10.1021/ja01600a058

6. Cava, M. P.; Levinson, M. I. Tetrahedron 1985, 41, 5061-5087. http://dx.doi.org/10.1016/S0040-4020(01)96753-5

7. Cherkasov, R. A.; Kutyrev, G. A.; Pudovik, A. N. Tetrahedron 1985, 41, 2567-2569. http://dx.doi.org/10.1016/S0040-4020(01)96363-X

8. Jones, B. A.; Bradshaw, J. S. Chem. Rev. 1984, 84, 17-30. http://dx.doi.org/10.1021/cr00059a002

9. Ozturk, T.; Ertas, E.; Mert, O. Chem. Rev. 2007, 107, 5210-5278. http://dx.doi.org/10.1021/cr040650b

10. Ozturk, T.; Ertas, E.; Mert, O. Chem. Rev. 2010, 110, 3419-3478. http://dx.doi.org/10.1021/cr900243d

11. Philip, J. W.; Chivers, T. Inorg. Chem. 2013, 52, 7791-7804.

http://dx.doi.org/10.1021/ic401002b

12. Hua, G.; Du, J.; Surgenor, B. A.; Slawin, A. M. Z.; Woollins, J. D. Molecules 2015, 20, 12175-12197. http://dx.doi.org/10.3390/molecules200712175

13. van Zyl, W. E.; Woollins, J. D. Coord. Chem. Rev. 2013, 257, 718-731.

http://dx.doi.org/10.1016/j.ccr.2012.10.010

14. Gray, I. P., Milton, H. L., Slawin, A. M. Z.; Woollins, J. D. Phosphorus, Sulfur, and Silicon and the Related Elements 2004, 179, 969-970.

http://dx.doi.org/10.1080/10426500490429158

15. Gray, I. P., Slawin, A. M. Z.; Woollins, J. D. Z. Anorg. Allgem. Chem. 2004, 630, 1851-1857. http://dx.doi.org/10.1002/zaac.200400161

16. Gray, I. P., Milton, H. L., Slawin, A. M. Z.; Woollins, J. D. Dalton Trans. 2003, 3450-3457. http://dx.doi.org/10.1039/b306387a

17. Hua, G.; Henry, J. B.; Li, Y.; Mount, A. R., Slawin, A. M. Z.; Woollins, J. D. Org. Biomol. Chem. 2010, 8, 1655-1660.

http://dx.doi.org/10.1039/b924986a

18. Hua, G.; Woollins, J. D. Angew. Chem., Int. Ed. 2009, 48, 1368-1377.

http://dx.doi.org/10.1002/anie.200800572

19. Hua, G.; Li, Y.; Fuller, A. L.; Slawin, A. M. Z.; Woollins, J. D. Eur. J. Org. Chem. 2009, 1612-1618. http://dx.doi.org/10.1002/ejoc.200900013

20. Hua, G.; Fuller, A. L.; Li, Y.; Slawin, A. M. Z.; Woollins, J. D. New J. Chem. 2010, 34, 1565-571. http://dx.doi.org/10.1039/b9nj00570f

21. Hua, G.; Fuller, A. L.; Li, Y.; Slawin, A. M. Z.; Woollins, J. D. Eur. J. Org. Chem. 2010, 2707-2615.

22. Hua, G.; Fuller, A. L.; Li, Y.; Slawin, A. M. Z.; Woollins, J. D. Polyhedron 2011, 30, 805-808. http://dx.doi.org/10.1016/j.poly.2010.12.018

23. Hua, G.; Fuller, A. L.; Li, Y.; Slawin, A. M. Z.; Woollins, J. D. Eur. J. Org. Chem. 2011, 3067-3073. http://dx.doi.org/10.1002/ejoc.201100226 
24. Hua, G.; Griffin, J. M.; Ashbrook, S. E.; Slawin, A. M. Z.; Woollins, J. D. Angew. Chem. Int. Ed. 2011, 50, 4123-4126.

http://dx.doi.org/10.1002/anie.201006081

25. Hua, G.; Cordes, D. B.; Li, Y.; Slawin, A. M. Z.; Woollins, J. D. Tetrahedron Lett. 2011, 52, 3311-3314.

http://dx.doi.org/10.1016/j.tetlet.2011.04.058

26. Hua, G.; Slawin, A. M. Z.; Randall, R. A. M.; Corde, D. B.; Crawford, L.; Bühl, M.; Woollins, J. D. Chem. Commun. 2013, 49, 2619-2621.

http://dx.doi.org/10.1039/c3cc40515j

27. Hua, G.; Du, J.; Corde, D. B.; Slawin, A. M. Z.; Woollins, J. D. J. Org. Chem. 2016, 81, 4210-4225. http://dx.doi.org/10.1021/acs.joc.6b00573

28. Hua, G.; Du, J.; Slawin, A. M. Z.; Woollins, J. D.Chem. Eur. J. 2016, 22, 7782-7791. http://dx.doi.org/10.1002/chem.201504832

29. Foreman, M. R. St. J.; Slawin, A. M. Z.; Woollins, J. D. J. Chem. Soc. Dalton Trans. 1996, 3653-3657. http://dx.doi.org/10.1039/dt9960003653

30. Parveen, S.; Kilian, P.; Slawin, A. M. Z.; Woollins, J. D. Dalton Trans. 2006, 2586-2590. http://dx.doi.org/10.1039/B517093A

31. Gray, I. P.; Slawin, A. M. Z.; Woollins, J. D. New J. Chem. 2004, 28, 1383-1389. http://dx.doi.org/10.1039/b406007e

32. Allen, F. H.; Kennard, O. Chem. Des. Autom. News 1993, 8, 1-31.

33. Lee, J. D.; Goodacre, G. W. Acta Crystallogr. Sect. B: Struct. Crystallogr. Cryst.Chem. 1971, 27, $1841-1845$. http://dx.doi.org/10.1107/S0567740871004953

34. John Foreman, M. R. St.; Slawin, A. M. Z.; Woollins, J. D. J. Chem. Soc. Dalton Trans. 1999, 1175-1184. http://dx.doi.org/10.1039/a808918c

35. Schwarz, P.; Ohms, G.; Kruger, K.; Grossmann, G.; Kaiser, V. Phosphorus, Sulfur, Silicon, Relat. Elem. 1998, 141, 27-43.

http://dx.doi.org/10.1080/10426509808033718

36. Wrackmeyer, B.; Klimkina, E. V.; Milius, W. Dalton Trans. 2014, 43, 5021-5043.

http://dx.doi.org/10.1039/C3DT52753K

37. Mandell, C. L.; Kleinbach, S. S.; G.Dougherty, W.; Kassel, W. S.; Nataro, C. Inorg. Chem. 2010, 49, 97189727.

http://dx.doi.org/10.1021/ic1016164

38. Sheldrick, G. M. Acta Cryst. 2008, A64, 112-122.

http://dx.doi.org/10.1107/S0108767307043930

39. CrystalClear-SM Expert v2.0 and v2.1. Rigaku Americas, The Woodlands, Texas, USA, and Rigaku Corporation, Tokyo, Japan, 2010-2013.

40. Palatinus, L.; Chapuis, G. J. Appl. Cryst. 2007, 40, 786-790.

http://dx.doi.org/10.1107/S0021889807029238

41. Burla, M. C.; Caliandro, R.; Camalli, M.; Carrozzini, B.; Cascarano, G. L.; Giacovazzo, C.; Mallamo, M.; Mazzone, A.; Polidori, G.; Spagna, R. J. Appl. Cryst. 2012, 45, 357-361.

http://dx.doi.org/10.1107/S0021889812001124

42. Sheldrick, G. M. Acta Crystallogr., Sect. C. 2015, 71, 3-8.

http://dx.doi.org/10.1107/S2053229614024218

43. DIRDIF-99. Beurskens, P. T.; Beurskens, G.; de Gelder, R.; Garcia-Granda, S.; Gould, R. O.; Israel, R.; Smits, J. M. M. Crystallography Laboratory, University of Nijmegen, The Netherlands, 1999. 
44. CrystalStructure v4.1. Rigaku Americas, The Woodlands, Texas, USA, and Rigaku Corporation, Tokyo, Japan, 2013. 